\title{
Enhanced Survival in Locoregionally Confined Anaplastic Thyroid Carcinoma: A Single-Institution Experience Using Aggressive Multimodal Therapy
}

\author{
Robert L. Foote, ${ }^{1,2}$ Julian R. Molina, ${ }^{1,3}$ Jan L. Kasperbauer,, ${ }^{1,4}$ Ricardo V. Lloyd, ${ }^{1,5}$ Bryan Mclver,, \\ John C. Morris, ${ }^{1,6}$ Clive S. Grant, ${ }^{1,7}$ Geoffrey B. Thompson,, ${ }^{1,7}$ Melanie L. Richards, ${ }^{1,7}$ \\ Ian D. Hay, ${ }^{1,6}$ Robert C. Smallridge, ${ }^{1,8}$ and Keith C. Bible ${ }^{1,3}$
}

Background: Historical outcomes in anaplastic thyroid carcinoma (ATC) are poor, with a median survival of only 5 months and $<20 \%$ of patients surviving 1 year from diagnosis. We hypothesized that survival in newly diagnosed patients with stages IVA and IVB locoregionally confined ATC might be improved by utilizing an aggressive therapeutic approach, prioritizing both the eradication of disease in the neck and preemptive treatment of occult metastatic disease.

Methods: Between January 1, 2003, and December 31, 2007, 25 new ATC patients were evaluated at our institution. Of these 25 patients, $10(40 \%)$ had metastatic disease at diagnosis and therefore underwent palliative treatment, whereas $5(20 \%)$ had regionally confined disease and desired treatment at their local medical facilities. The remaining 10 consecutive patients (40\%) had regionally confined ATC and elected aggressive therapy combining individualized surgery (where feasible), intensity-modulated radiation therapy (IMRT), and radiosensitizing + adjuvant chemotherapy intending four cycles of docetaxel + doxorubicin. Outcomes were assessed on an intention to treat basis.

Results: There were no deaths from therapy, but hospitalization was required in two patients (20\%) because of treatment-related adverse events. Five patients $(50 \%)$ are alive and cancer-free, all having been followed $>32$ months (range: 32-89 months; median: 44 months) with a median overall Kaplan-Meier survival of 60 months. Overall survival at 1 and 2 years was $70 \%$ and $60 \%$, respectively, compared to $<20 \%$ historical survival at 1 year in analogous patients previously treated with surgery and conventional postoperative radiation at our and other institutions.

Conclusions: Although based upon a small series of consecutively treated patients, an aggressive approach combining IMRT and radiosensitizing plus adjuvant chemotherapy appears to improve outcomes, including survival in stages IVA and IVB regionally confined ATC, but remains of uncertain benefit in patients with stage IVC (metastatic) disease. Also uncertain is the optimal chemotherapy regimen to use in conjunction with IMRT. Further multicenter randomized trials are required to define optimal therapy in this rare but deadly cancer.

\section{Introduction}

A NAPLASTIC THYROID CARCINOMA (ATC) is a malignancy with terrible prognosis-only $20 \%$ of affected patients survive 1 year from diagnosis, and median survival is only 5 months (1). Compounding therapeutic progress in ATC is its rarity. In the 50-year period between 1949 and 1999 at our institution, on average only two to three new ATC patients were seen annually (2), making it extremely challenging to develop new treatment approaches. Not surprisingly, therefore, no randomized therapeutic clinical trials have yet been accomplished in ATC.

In reviewing historical data at our institution seeking identification of approaches that might be implemented to improve ATC outcomes, we noted that among 134 new ATC patients treated at Mayo in our original 50-year series, 13 received combined modality therapy including surgical debulking, postoperative radiation therapy, and doxorubicin

\footnotetext{
${ }^{1}$ Endocrine Malignancies Disease Oriented Group, Mayo Clinic Comprehensive Cancer Center, Rochester, Minnesota.

${ }^{2}$ Department of Radiation Oncology, ${ }^{3}$ Division of Medical Oncology, ${ }^{4}$ Department of Otorhinolaryngology, ${ }^{5}$ Department of Laboratory Medicine and Pathology, ${ }^{6}$ Division of Endocrinology, and ${ }^{7}$ Department of Surgery, Mayo Clinic, Rochester, Minnesota.

${ }^{8}$ Division of Endocrinology, Mayo Clinic, Jacksonville, Florida.
} 
radiosensitizing chemotherapy (2). Although median survival among these patients did not differ significantly from the group as a whole or from those receiving conventional postoperative radiation therapy alone, a greater proportion of patients survived longer than 1 year $(23 \%$ vs. $<10 \%)$ - suggesting perhaps that systemic chemotherapy might have potential to treat micrometastatic disease and affect improved long-term survival. Our experience had also been that almost all patients succumb to metastatic disease even if disease could be controlled in the neck, so it was clear to us that ATC in most all patients is a systemic disease - even at the time of initial diagnosis. Moreover, it was disheartening to find in our original series that conventional external beam radiation therapy did not alter the chances of locoregional tumor recurrence, although it did appear to somewhat delay its development (2).

We therefore postulated that to make progress and extend survival in ATC, a two-pronged approach would be required to both more effectively treat locoregional disease while in parallel preemptively aggressively treating occult metastatic disease. Hence, beginning in 2003, patients with operable or inoperable nonmetastatic ATC (stages IV A and B) who desired an aggressive approach were routinely offered and treated with individualized intensity modulated radiation therapy (IMRT) and radiosensitizing as well as adjuvant chemotherapy. IMRT was utilized because of its more favorable dose distributions, allowing optimal dose delivery to the target volume while minimizing dose to uninvolved organs including the larynx, esophagus, and lung (3-6). Doxorubicin was chosen as one of the chemotherapeutic agents to be utilized because of its known activity in advanced ATC and its radiation sensitizing properties (7-15). A taxane, either docetaxel or paclitaxel, was also chosen to be added to the doxorubicin and radiation therapy because of reported promising activity against advanced ATC (16), the established synergy between taxanes and doxorubicin $(17,18)$ and a known safety profile with the administration of the combination of doxorubicin, a taxane, and radiation therapy $(18,19)$. The present report details the long-term outcomes for the first 10 consecutive patients treated in accord with these revised practice guidelines.

\section{Methods}

This retrospective analysis analyzing outcomes from our practice change was approved by the Mayo Clinic Institu- tional Review Board. In accordance with Minnesota state law, only patients who authorized review of their medical records for research were included in this report. The medical diagnostic index and Cancer Registry, institutional databases listing all patient diagnoses, were searched electronically to identify all newly diagnosed patients with and/or treated for ATC between January 1, 2003, and December 31, 2007. Patients with undifferentiated thyroid cancers (grade $3 / 4$ ) not meeting review criteria for ATC (grade 4/4) were specifically excluded from this case series. January 2003 was chosen as the beginning date for the study because this is the date that IMRT for thyroid cancer was introduced into our clinical practice. A total of 25 new ATC patients were identified in this 5-year interval: $10(40 \%)$ presented with distant metastatic disease and underwent palliative treatment, whereas 5 (20\%) presenting with regionally confined disease elected treatment at their local institutions (Scheme 1).

Of the remaining 10 consecutive patients $(40 \%$ of all new ATC patients seen at our institution) all had regionally confined ATC, all were of good performance status, and all were offered an aggressive approach in accord with our altered practice guidelines. All 10 elected to proceed with treatment using IMRT and concurrent chemotherapy at Mayo Clinic (9) or at another institution under the direction of Mayo Clinic physicians (1). All 10 patients had tissue confirmation of ATC by expert thyroid pathologists at our institution. For 8 cases, pathological material was available for confirmatory rereview conducted by a single expert pathologist (R.V.L.). None of the 10 patients had evidence of distant metastatic disease (stage IVC) based on physical examination, routine labs, and imaging including computed tomography (CT) scans of the chest and abdomen, and positron emission tomography (PET)/CT. After treatment, patients were usually seen for follow-up every 3 months for the first 2 years, then every 6 months for an additional 3 years. Median follow-up was 36 months.

Our intention was to utilize radiosensitizing chemotherapy as well as several additional cycles of postradiation therapy and/or preradiation chemotherapy, to consist of doxorubicin combined with docetaxel (day 1: docetaxel $60 \mathrm{mg} / \mathrm{m}^{2}$ and doxorubicin $60 \mathrm{mg} / \mathrm{m}^{2}$ administered intravenously; day 2: pegylated Filgrastim, $6 \mathrm{mg}$ subcutaneously administered $>24$ hours after completion of chemotherapy, all administered on an outpatient basis) (18). Individualization of this chemotherapy regimen guideline, however, was allowed at the discretion

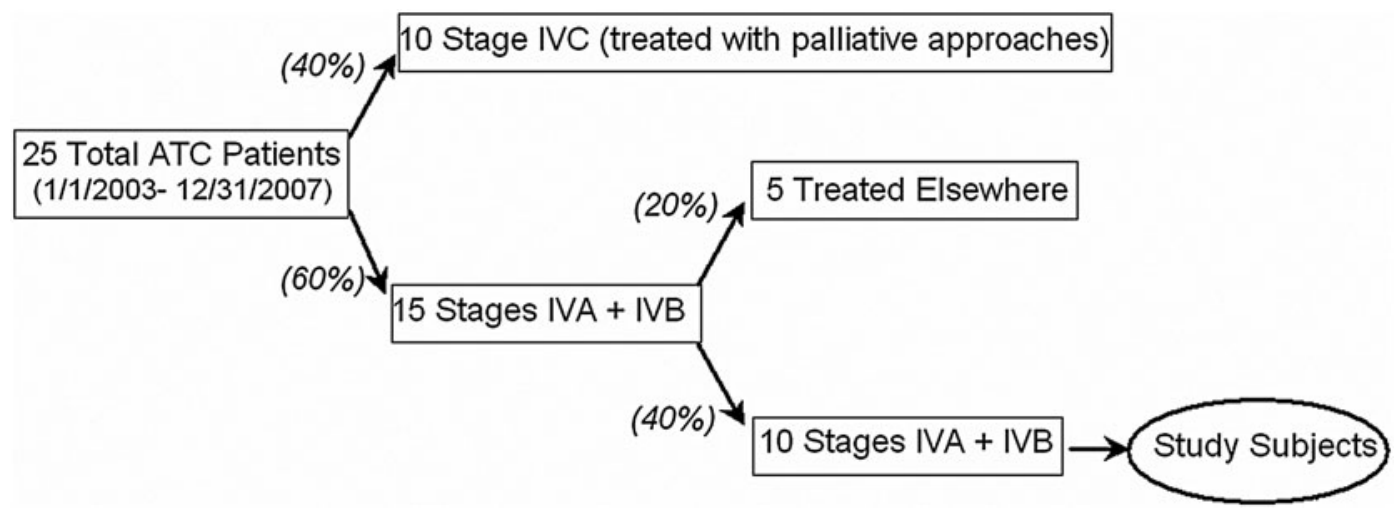

SCHEME 1. Distribution of ATC Patients seen during study period. 
Table 1. Overall Patient Characteristics and Chemotherapy Agents Utilized

\section{Patient characteristics}

Age in years: median (range)

Gender (male:female)

$57(45-83)$

Stage (IVA:IVB)

Co-existing differentiated thyroid carcinoma (yes:no)

Extent of surgical resection (R0:R1:R2)

Chemotherapy administered (number of patients)

Doxorubicin

Taxane (paclitaxel or docetaxel)

Platin (cisplatin or carboplatin)
$7: 3$

$7: 3$

$4: 6$

$4: 3: 3$ of treating physicians so as to permit modification in response to patient medical history (e.g., impaired cardiac ejection fraction and preexisting neuropathy) as well as patient tolerance. Consequently, heterogeneity in the administered chemotherapy necessarily resulted. Patients received agents as shown in Table 1, with $7 / 10(70 \%)$ receiving multi-agent chemotherapy, but with only $5 / 10(50 \%)$ receiving docetaxel and doxorubicin combination chemotherapy as originally intended. Nonetheless, data from all 10 patients are included in this report using an intention to treat analysis despite this heterogeneity resulting from individualization of chemotherapy.

The target volume for IMRT was to include any residual cancer within the thyroid bed and/or regional lymph nodes, with elective treatment of bilateral level II through VI and mediastinal lymph nodes to the level of the carina. Like chemotherapy, individualization of radiotherapy was permitted. Nine or 10 fields were utilized with dynamic multi-leaf collimators. Overall survival estimates were calculated using the KaplanMeier method from the date of pathologic diagnosis of ATC.
In our approach, we deliberately elected to group patients by tumor stage (IVA and IVB vs. IVC) rather than by extent of resection. This decision was based upon our prior data indicating that, while tumor stage was as strong predictor of survival, extent of resection was not (2). Consequently, we did not eliminate consideration of intended aggressive multimodal therapy based upon incomplete (R1 or R2) resection or tumor regrowth in the time prior to institution of IMRT.

\section{Results}

Patient characteristics are summarized in Table 1, with specific individual patient data provided in Table 2 . Seven men and three women were treated; median age was 57 years (range: 45-83 years). Confirmatory pathological re-review of the $8 / 10$ available specimens combined with examination of pathological records confirmed ATC in the absence of background differentiated thyroid cancer in six, ATC arising in a background of papillary thyroid carcinoma in two, ATC arising in a background of follicular thyroid carcinoma in one, and ATC arising with papillary thyroid carcinoma and squamous cell carcinoma in one. In all four cases of mixed histology, the anaplastic component was dominant, constituting a major component to the primary tumors and not an incidentally noted ATC focus. Metastatic disease was excluded by imaging in all patients; CT scans of the chest were obtained in 8/10 (including abdomen in six) and PET/CT was additionally obtained in $9 / 10$ patients. All 10 patients were treated with curative intent.

Surgical intervention included complete resection in four patients, incomplete resection in five patients (microscopically positive margins in three, debulking procedures with gross residual disease in two), and biopsy only in one patient. Of the four patients undergoing complete surgical resection, one developed a local-regional recurrence in the interval between surgery and initiation of chemotherapy and radiation therapy, thereby leaving only 3 of 10 patients $(30 \%)$

Table 2. Specific Patient Characteristics, Therapies, Adverse Effects and Outcomes

\begin{tabular}{|c|c|c|c|c|c|c|c|c|c|c|}
\hline Pt. & Age & Gender & $\begin{array}{l}\text { Extent of } \\
\text { resection }\end{array}$ & $\begin{array}{l}\text { Extent of } \\
\text { disease }\end{array}$ & $\begin{array}{l}\text { Radiation } \\
\text { therapy }\end{array}$ & Chemotherapy & $\begin{array}{l}\text { Adverse } \\
\text { effects }\end{array}$ & OS & TTP & $\begin{array}{l}\text { Site of first } \\
\text { progression }\end{array}$ \\
\hline 1 & 45.5 & Male & R0 & LR & ABID, 64 Gy & Con. AT & None & 3.7 & 1.4 & $\mathrm{DM}$ \\
\hline 2 & 55.4 & Male & R0 & NED & SB, 66 Gy & Con. A & None & 52.5 & NA & None \\
\hline 3 & 58.4 & Male & $\mathrm{R} 2$ & LR & SB, 61.84Gy & Con. A & PEG & 67.9 & 22.8 & LRR \\
\hline 4 & 62.1 & Male & R1 & LR & SB, 66 Gy & Con. T,C & $\begin{array}{l}\text { DVT, PE, } \\
\text { Tater, Hosp. }\end{array}$ & 40.5 & NA & None \\
\hline 5 & 52.2 & Male & R0 & NED & SB, $64.5 \mathrm{~Gy}$ & Con. PCb & None & 7.03 & 2.6 & LRR, DM \\
\hline 6 & 62.6 & Female & $\mathrm{R} 2$ & LR & C, $70 \mathrm{~Gy}$ & Con. AT & $\begin{array}{l}\text { ES, Stiff neck, } \\
\text { Hosp. }\end{array}$ & 32.2 & NA & None \\
\hline 7 & 58.3 & Male & R1 & LR & C, $70 \mathrm{~Gy}$ & Con. C Adj. AT & None & 22.1 & 2.38 & LRR, DM \\
\hline 8 & 55.7 & Male & $\mathrm{R} 2$ & LR & ABID, 60 Gy & Con. AT & $\begin{array}{l}\text { HC, Pneum., } \\
\text { FTT }\end{array}$ & 10.1 & 4.1 & $\mathrm{DM}$ \\
\hline 9 & 83.5 & Female & R1 & LR & ABID, 57.6 Gy & Con. A & None & 89.3 & NA & None \\
\hline 10 & 48.4 & Female & R0 & NED & C, 59.4Gy & $\begin{array}{l}\text { Ind. AT Con. } \\
\text { PCb Adj. AT }\end{array}$ & None & 44.4 & NA & None \\
\hline
\end{tabular}

Pt., patient (age in years); OS, overall survival in months; TTP, time to progression in months; NA, not applicable; R0, no residual tumor; R1, microscopic residual tumor; R2, macroscopic residual tumor; NED, no evidence of disease; LR, local-regional disease; ABID, accelerated twice-a-day; SB, simultaneous boos; C, conventional; Gy, Gray; Con., concurrent; A, doxorubicin; T, docetaxel; C, cisplatin; P, paclitaxel; Cb, carboplatin; Ind., induction; Adj., adjuvant; PEG, percutaneous enteral gastrostomy tube; DVT, deep venous thrombosis; PE, pulmonary embolism; Tater, reaction to docetaxel; ES, esophageal stenosis; Hosp., hospitalized during treatment; HC, hemorrhagic cystitis; Pneum., grade 3 pneumonitis; FTT, failure to thrive; DM, distant metastasis; LRR, local regional recurrence. 
pathologically free of disease at times of initiation of combined IMRT and chemotherapy.

The median time interval from the date of surgery (or biopsy in the case of one patient) to the date of the first chemotherapy was 23 days (range: 9 to 51 days). Radiation therapy consisted of accelerated, twice-daily IMRT in three, once-daily slightly accelerated simultaneous integrated boost IMRT in four, and once-daily conventional fractionation IMRT in three. The median time interval from the date of the first chemotherapy administration to the date of first radiation therapy treatment was 0 days, with a range of the chemotherapy beginning 28 days before the radiation therapy to 2 days after the first day of radiation therapy. The median overall radiation therapy treatment time was 42 days (range: 23 to 49 days); median dose per fraction was 2.0 Gy (range: 1.5 to $2.2 \mathrm{~Gy}$ ). The median number of fractions was 34 (range: 29 to 40 fractions), and the median total dose was $64.25 \mathrm{~Gy}$ (range: 57.6 to $70 \mathrm{~Gy}$ ). The median time interval from the date of surgery or biopsy to the first day of radiation therapy was 28 days (range: 9 to 51 days).

Long-term locoregional tumor control was achieved in 7 of 10 treated patients. One patient who developed local and regional recurrence died with local, regional, and distant disease 68 months after the original diagnosis and 45 months after a course of re-irradiation utilizing hyperfractionated IMRT (60 Gy in 50 fractions, 1.2 Gy per fraction, over 6 weeks) and concurrent weekly carboplatin and paclitaxel (six cycles).

Median follow-up for all patients was 36 months (range: 4 to 89 months), with a median overall Kaplan-Meier survival of 60 months (shown in Fig. 1 compared to 50-year Mayo Clinic historical outcome data for stages IVA, IVB, and IVC ATC). At last follow-up, five patients (50\%) were alive having been followed for a minimum of 32 months (range: 32-89 months; median: 44 months) and actual 1- and 2-year overall survival was $70 \%$ and $60 \%$, respectively. The five deaths occurred 4, 7, 10, 22, and 68 months after initial ATC diagnosiswith distant metastases contributory in all five, and with three also involving locoregional recurrences.

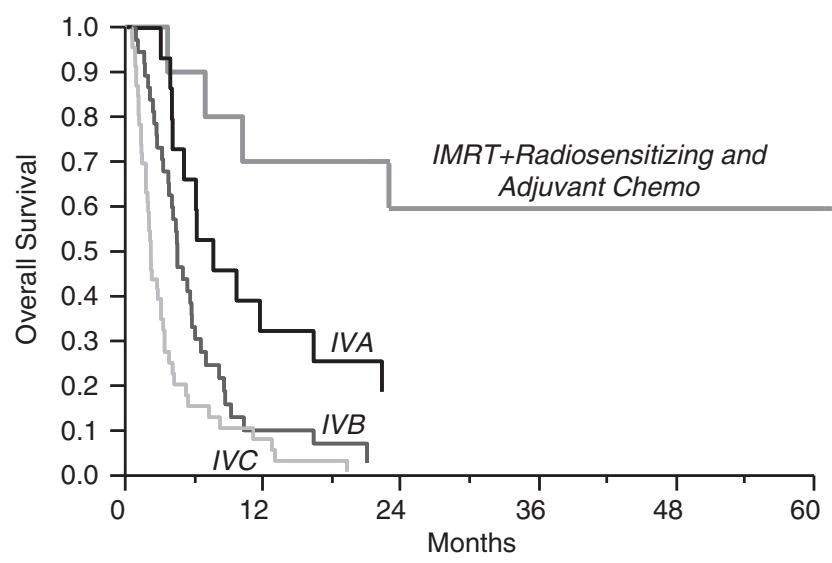

FIG. 1. Overall Kaplan-Meier survival in months. Data for historical 50-year Mayo Clinic outcomes for stages IVA, IVB, and IVC anaplastic thyroid cancer are compared to outcomes resulting from our recent practice change combining intensity modulated radiation therapy with adjuvant and radiosensitizing chemotherapy. IMRT, intensity modulated radiation therapy.
There were no treatment-related deaths. Morbidity observed during therapy included one patient who developed a self-limited reaction to docetaxel consisting of hypertension, muscle spasms, pelvic pain, diffuse erythema of the skin, and anxiety that prohibited subsequent taxane use. Another patient treated with doxorubicin and docetaxel developed selflimited acute hemorrhagic cystitis. One patient developed bilateral deep venous thrombosis of the lower extremities and a pulmonary embolism, requiring placement of an inferior vena cava filter and anticoagulation during treatment. Two of the 10 patients $(20 \%)$ required hospitalization during concurrent chemo- and radiation therapy, one for a pulmonary embolism and the other on two occasions, one for nausea and vomiting early on in treatment and then again near the end of treatment for percutaneous endoscopic gastrostomy tube placement, both 1-2-day admissions.

Late adverse effects of therapy and/or disease include one patient who became both tracheostomy and feeding tube dependent; one patient who developed grade 3 pneumonitis (Common Terminology Criteria for Adverse Events, CTCAE, Version 3.0), which subsequently resolved; one patient who developed failure-to-thrive, which subsequently resolved; two patients who developed esophageal stenosis, one of which resolved with dilatation; and one patient who was noted to have a stiff, fibrotic neck that remains symptomatic but that does not significantly affect activities of daily living or employment.

\section{Discussion}

Historically, the diagnosis of ATC has been almost uniformly a death sentence, with a median survival of 5 months and only $20 \%$ survival 1 year from diagnosis (1-3). ATC confers among the worst prognosis of any cancer, considerably worse overall than lung cancer, renal cell carcinoma, or even pancreatic cancer. Surveillance, Epidemiology, and End Results data indicate a 2- and 5-year survival of $32.7 \%$ and $22.9 \%$, respectively, even when the cancer is confined entirely to the thyroid gland, declining to $16.1 \%$ and $10.1 \%$, respectively, in the presence of extracapsular extension; with more extensive disease, including distant metastases, 2-year survival falls to $2.1 \%$ (20).

The present report indicating 70\% 1-year and 60\% 2-year survival-and 50\% >2.5-year disease free survival with a median overall Kaplan-Meier survival of 5 years-is therefore extremely encouraging, and represents an apparent improvement over the 4-month median survival (and superimposed survival curves) we had previously observed in patients treated historically with external beam radiation therapy and doxorubicin radiosensitization (2). Although the number of patients in this series is modest, we believe the results from our pilot approach to be highly promising.

As to why outcomes in the present series may be superior to prior/historical multimodal outcomes, interval "innovations" that may be contributory include (i) the advent of IMRT, (ii) the addition of taxane chemotherapy (to prior anthracyclineonly therapy), and (iii) the use of adjuvant chemotherapy cycles in addition to only that required for radiosensitization. As we undertook all three in tandem in the present intervention, it is impossible to separate out individual versus combined influences of the three. What seems clear, however, is that these innovations appear collectively to have produced superior results to those previously attained. 
It is important to acknowledge, however, that outcomes in our series may be favorably biased by several factors. First, as patients with overt metastatic disease were excluded, the present data are relevant only to patients with stages IVA and IVB locoregionally confined disease at diagnosis. Indeed, we have not been able to effectively salvage patients with metastatic disease using the present approach. Also, as participants both chose to follow an aggressive therapeutic approach and were deemed capable of withstanding such an approach, selection bias may contribute to better outcomes. However, we report outcomes in 10 consecutively treated patients-with none excluded-so as to minimize further confounding factors that might otherwise contribute to biased outcomes. Moreover, these same biases existed in the series of 13 patients we had historically treated with external beam radiation therapy and doxorubicin that demonstrated no survival advantage (2).

Although there was individualization, and therefore heterogeneity, of both IMRT and chemotherapy, all 10 consecutively treated patients were included in this report, as we elected an intention to treat analysis. This report also represents a case series analyzing results from our practice change rather than a prospective clinical trial; however, given the rarity of ATC and historically dire prognosis, mounting a formal clinical trial in the absence of pilot data proved untenable. Indeed, consistent with our experience and to the best of our knowledge no prospective randomized clinical trial has yet been conducted in this patient population.

It is noteworthy also that several recent reports in addition to our own bolster the hypothesis that the addition of both radiosensitizing and adjuvant chemotherapy to surgery and radiotherapy may improve ATC outcomes. Among 30 ATC patients treated with surgery, radiation therapy and doxorubicin administered concurrently with radiation therapy and continued thereafter, Swaak-Kragten et al. reported an improvement in 1-year survival from $9 \%$ to $23 \%$ (21). Haigh et al. reported $75 \%$ 2-year survival in eight patients undergoing complete surgical resection and adjuvant radiation therapy and chemotherapy (22). Tan et al. reported 5-year survival in three of five patients who underwent complete resection, four receiving postoperative radiation therapy and three receiving postradiation doxorubicin chemotherapy (23). Pudney et al. reported a median survival of 13 months for five patients with stage IVB ATC treated with either radiation therapy concurrent with doxorubicin or with induction docetaxel, doxorubicin and cyclophosphamide (24). Similarly, Higashiyama et al. reported 44\% 1-year survival in nine patients with stage IVB ATC treated with induction paclitaxel in comparison to $5.9 \%$ in a historical control group (25).

Despite these pilot data intimating improved ATC outcome from aggressive up-front therapy, several critical questions remain that should ideally be addressed in multicenter randomized trials. In particular, although the early integration of systemic therapy of various kinds in patients with stages IVA and IVB ATC appears to result in improved outcomes, including survival, it is still unclear as to which systemic therapy is best. In our series, there was no clear correlation between the chemotherapy administered and outcome, leaving the impression that some chemotherapy was better than no chemotherapy, but leaving the question of optimal approach still unresolved.
Similar uncertainty remains about the optimal means of administration of radiotherapy. We have chosen to utilize IMRT, as it allows us to administer a more conformal high dose (60 to $70 \mathrm{~Gy}$ ) with improved homogeneity to the gross disease and high-risk areas (lymph nodes and tracheoesophageal groove) while lowering the dose to normal organs at risk, including the salivary glands, epiglottis, false vocal cords, true vocal cords, arytenoids, central and posterior aspects of the pharyngeal constrictors and esophagus, brachial plexus, and spinal cord. The optimal fractionation schedule, however, has not yet been determined. Moderate dose (57.6 Gy), twice-daily, 3 days per week, slightly accelerated radiation therapy combined with concurrent weekly doxorubicin required interruption in $70 \%$ of patients due to severe pharyngoesophagitis and tracheitis (10). High-dose (60.8 Gy)accelerated fractionation even without concurrent chemotherapy may be too toxic, as resulting median overall survival using this approach in ATC was reported to be just 10 weeks (26). Low-dose (40 Gy)-hyperfractionated-accelerated radiation therapy with sequential but not concurrent chemotherapy (doxorubicin) appears, however, to be well tolerated and effective in ATC, with $50 \%$ and $35 \% 1$ and 3 years; survival, respectively (27). High-dose (60 Gy)-hyperfractionatedaccelerated radiation therapy alone in regionally confined ATC appears well tolerated, but with a median overall survival of just 13.6 months in one series (28), and $<10 \% 1$ year survival in another (29), consistent with our hypothesis that the addition of systemic therapy may be critical to achieving improved long-term survival.

With regard to the question of effectiveness of locoregional control, two of the three patients who developed locoregional recurrence in our series were treated with once-daily, slightly accelerated, simultaneous integrated boost IMRT using fraction sizes of 2.16 and 2.15Gy to total doses of 61.84 and $64.5 \mathrm{~Gy}$, respectively. One of these patients was a long-term survivor following hyperfractionated re-irradiation with IMRT to 60 Gy using 1.2 Gy per fraction twice-daily. The third patient with a local-regional recurrence was treated with once-daily conventional fractionation IMRT consisting of 35 fractions of $2 \mathrm{~Gy}$ each for a total dose of $70 \mathrm{~Gy}$. There were no local-regional recurrences in the three patients in our series treated with accelerated twice-daily IMRT, raising the possibility of superior local control using the twice-daily accelerated approach.

In summary, our consecutive series of stage IVA and IVB ATC patients treated with IMRT and aggressive combination chemotherapy demonstrates encouraging long-term survival with acceptable, although significant, toxicity. We believe that these data, combined with encouraging pilot reports at other centers, provide a compelling rationale for further investigation of this approach to treating a disease that has historically been otherwise met with very discouraging outcomes.

\section{Acknowledgment}

The authors would like to thank and acknowledge Vera J. Suman, Ph.D., Cancer Center Statistics, Mayo Clinic Rochester, for her editorial review of the article.

\section{Disclosure Statement}

The authors have nothing to disclose. 


\section{References}

1. Smallridge RC 2010 Anaplastic thyroid carcinoma: pathogenesis and emerging therapies. Clin Oncol (R Coll Radiol) 22:486-497.

2. McIver B, Hay ID, Giuffrida DF, Dvorak CE, Grant CS, Thompson GB, van Heerden JA, Goellner JR 2001 Anaplastic thyroid carcinoma: a 50-year experience at a single institution. Surgery 130:1028-1034.

3. Nutting CM, Convery DJ, Cosgrove VP, Rowbotton C, Vini L, Harmer C, Dearnaley DP, Webb S 2001 Improvements in target coverage and reduced spinal cord irradiation using intensity-modulated radiotherapy (IMRT) in patients with carcinoma of the thyroid gland. Radiother Oncol 60:173-180.

4. Urbano TG, Clark CH, Hansen VN, Adams EJ, Miles EA, Mc Nair H, Bidmead AM, Warrington J, Dearnaley DP, Harmer C, Harrington KJ, Nutting CM 2007 Intensity modulated radiotherapy (IMRT) in locally advanced thyroid cancer: acute toxicity results of a phase I study. Radiother Oncol 85:58-63.

5. Rosenbluth BD, Serrano V, Happersett L, Shaha AR, Tuttle RM, Narayana A, Wolden SL, Rosenzweig KE, Chong LM, Lee Ny 2005 Intensity-modulated radiation therapy for the treatment of nonanaplastic thyroid cancer. Int J Radiat Oncol Biol Phys 63:1419-1426.

6. Schwartz DL, Lobo MJ, Ang KK, Morrison WH, Rosenthal DI, Ahamad A, Evans DB, Clayman G, Sherman SI, Garden AS 2009 Postoperative external beam radiotherapy for differentiated thyroid cancer: outcomes and morbidity with conformal treatment. Int J Radiat Oncol Biol Phys 74:1083-1091.

7. Poster DS, Bruno S, Penta J, Pina K, Catane R 1981 Current status of chemotherapy in the treatment of advanced carcinoma of the thyroid gland. Cancer Clin Trials 4:301-307.

8. Teicher BA, Lazo JS, Sartorelli AC 1981 Classification of antineoplastic agents by their selective toxicities toward oxygenated and hypoxic tumor cells. Cancer Res 41:73-81.

9. Durand RE 1976 Adriamycin: a possible indirect radiosensitizer of hypoxic tumor cells. Radiology 19:217-222.

10. Kim JH, Leeper RD 1983 Treatment of anaplastic giant and spindle cell carcinoma of the thyroid gland with combination adriamycin and radiation therapy. A new approach. Cancer 52:954-957.

11. Kim JH, Leeper RD 1983 Combination Adriamycin and radiation therapy for locally advanced carcinoma of the thyroid gland. Int J Radiat Oncol Biol Phys 9:565-567.

12. Kim JH, Leeper RD 1987 Treatment of locally advanced thyroid carcinoma with combination doxorubicin and radiation therapy. Cancer 60:2372-2375.

13. Tennvall J, Tallroth E, El Hassan A, Lundell G, Akerman B, Biörklund A, Blombren H, Löwhagen T, Wallin G 1990 Anaplastic thyroid carcinoma. Doxorubicin, hyperfractionated radiotherapy and surgery. Acta Oncologica 29: $1025-1028$.

14. Tennvall J, Lundell G, Hallquist A, Wahlberg P, Wallin G, Tibblin S 1994 Combined doxorubicin, hyperfractionated radiotherapy, and surgery in anaplastic thyroid carcinoma. Cancer 74:1348-1354.

15. Tennvall J, Lundell G, Wahlberg P, Bergenfelz A, Grimelius L, Akerman M, Hjelm Skog A-L, Wallin G 2002 Anaplastic thyroid carcinoma: three protocols combining doxorubicin, hyperfractionated radiotherapy and surgery. Br J Cancer 86:1848-1853.

16. Ain KB, Egorin MJ, DeSimone PA 2000 Treatment of anaplastic thyroid carcinoma with paclitaxel: phase 2 trial using ninety-six-hour infusion. Collaborative Anaplastic Thyroid Cancer Health Intervention Trials (CATCHIT) Group. Thyroid 10:587-594.

17. Budman DR, Calabro A 2002 In vitro search for synergy and antagonism: evaluation of docetaxel combinations in breast cancer cell lines. Breast Cancer Res Treat 74:41-46.

18. Sledge GW Jr 1995 Doxorubicin/paclitaxel combination chemotherapy for metastatic breast cancer: the Eastern Cooperative Oncology Group experience. Semin Oncol $22(5$ Suppl 12):123-125.

19. Sprano JA, O'Neill A, Schaefer PL, Falkson CI, Wood WC 2000 Phase II trial of doxorubicin and docetaxel plus GCSF in metastatic breast cancer: Eastern Cooperative Oncology Group Study E1196. J Clin Oncol 18:2369-2377.

20. Chen J, Tward JD, Shrieve DC, Hitchcock YJ 2008 Surgery and radiotherapy improves survival in patients with anaplastic thyroid carcinoma. Am J Clin Oncol 31:460-464.

21. Swaak-Kragten AT, de Wilt JH, Schmitz PI, Bontenbal M, Levendag PC 2009 Multimodality treatment for anaplastic thyroid carcinoma-Treatment outcome in 75 patients. Radiother Oncol 92:100-104.

22. Haigh PI, Ituarte PHG, Wu HS, Treseler PA, Posner MC, Quivey JM, Duh QY, Clark OH 2001 Completely resected anaplastic thyroid carcinoma combined with adjuvant chemotherapy and irradiation is associated with prolonged survival. Cancer 91:2335-2342.

23. Tan RK, Finley RK, Driscoll D, Bakamijian V, Hicks WL Jr, Shedd DP 1995 Anaplastic carcinoma of the thyroid: a 24year experience. Head Neck 17:41-48.

24. Pudney D, Lau H, Ruether JD, Falck V 2007 Clinical experience of the multimodality management of anaplastic thyroid cancer and literature review. Thyroid 17:1243-1250.

25. Higashiyama T, Ito $Y$, Hirokawa M, Fukushima M, Uruno T, Miya A, Matsuzuka F, Miyauchi A 2010 Induction chemotherapy with weekly paclitaxel administration for anaplastic thyroid carcinoma. Thyroid 20:7-14.

26. Mitchell G, Huddart R, Harmer C 1999 Phase II evaluation of high dose accelerated radiotherapy for anaplastic thyroid carcinoma. Radiother Oncol 50:33-38.

27. De Crevoisier R, Baudin E, Bachelot A, Leboulleux S, Travagli JP, Caillou B, Schlumberger M 2004 Combined treatment of anaplastic thyroid carcinoma with surgery, chemotherapy, and hyperfractionated accelerated external radiotherapy. Int J Radiat Oncol Biol Phys 60:1137-1143.

28. Wang Y, Tsang R, Asa S, Dickson B, Arenovich T, Brierley J 2006 Clinical outcome of anaplastic thyroid carcinoma treated with radiotherapy of once- and twice-daily fractionation regimens. Cancer 107:1786-1792.

29. Dandekar P, Harmer C, Barbachano Y, Rhys-Evans P, Harrington K, Nutting C, Newbold K 2009 Hyperfractionated accelerated radiotherapy (HART) for anaplastic thyroid carcinoma: toxicity and survival analysis. Int J Radiat Oncol Biol Phys 74:518-521.

Address correspondence to: Keith C. Bible, M.D., Ph.D. Division of Medical Oncology Mayo Clinic 200 First Street SW Rochester, MN 55905

E-mail: bible.keith@mayo.edu 